\title{
Solar wind induced magnetic field around the unmagnetized Earth
}

\author{
G. T. Birk ${ }^{1}$, H. Lesch ${ }^{1}$, and C. Konz ${ }^{2}$ \\ 1 Institut für Astronomie and Astrophysik, Universität München, Scheinerstr 1, 81679 München, Germany \\ 2 Max-Planck-Institute for Plasma Physics, Garching, Germany
}

Received 9 February 2004 / Accepted 28 April 2004

\begin{abstract}
The Earth is a planet with a dipolar magnetic field which is agitated by a magnetized plasma wind streaming from the Sun. The magnetic field shields the Earth's surface from penetrating high energy solar wind particles, as well as interstellar cosmic rays. The magnetic dipole has reversed sign some hundreds of times over the last 400 million years. These polarity reversals correspond to drastic breakdowns of the dynamo action. The question arises what the consequences for the Earth's atmosphere, climate, and, in particular, biosphere are. It is shown by kinematic estimates and three-dimensional plasma-neutral gas simulations that the solar wind can induce very fast a magnetic field in the previously completely unmagnetized Earth's ionosphere that is strong enough to protect Earth from cosmic radiations comparable to the case of an intact magnetic dynamo.
\end{abstract}

Key words. Earth - solar wind - solar-terrestrial relations - magnetic fields - magnetohydrodynamics

\section{Introduction}

Paleomagnetic records show that the magnetism of Earth has reversed itself hundreds of times over the last 400 million years (Valet \& Meynardier 1993; Juarez et al. 1998; Gee et al. 2000; Selkin \& Tauxe 2000; Valet 2003). In fact, geomagnetic polarity reversals represent the most dynamic feature of the Earth's magnetic field. The polarity reversals do not occur instantaneously. Rather, transition periods, that span some thousand years and are characterized by unstable varying magnetic fields with no clear shape, lay between the stable dipole field states. During the transition periods the magnetic field strength can drop well below $10 \%$ of the average value (Juarez et al. 1998; Guyodo \& Valet 1999; Selkin \& Tauxe 2000) which signifies a drastic breakdown of the Earth's dynamo. In the present time the magnetic south pole has wandered over more than $1100 \mathrm{~km}$ during the last 200 years. The strength of the Earth's field has decreased by $5 \%$ per century. This decrease is by far the fastest that has been verified since the last total field reversal, the end of the so-called Matuyama chron, 730000 years ago. Also, by statistical estimates the Earth's dynamo is overdue for a reversal. Thus, we have to expect a transition period characterized by a very small Earth's magnetic field in the near future. Since the Earth's dipole field provides us with a shield against cosmic rays and solar high-energy radiation one may wonder about the consequences for life on Earth. Also, having in mind that Mars lost the atmosphere almost completely after the final

Send offprint requests to: $\mathrm{G}$. T. Birk,

e-mail: birk@usm.uni-muenchen.de breakdown of the magnetic field (Luhmann \& Bauer 1992), one may speculate that stripping by the solar wind could alter the Earth's atmosphere. Severe climate changes could result. Interesting enough, during the last Brunhes-Matuyama reversal, no major changes in plant and animal life have been detected. This may be partly due to the fact that the atmospheric layers block some fraction of the cosmic radiation by scattering. On the other hand, the cosmogenic radionuclide production varies at least over the last 200000 years as function of short-term variations of the magnetic field (Frank 2000).

In this contribution. we consider the interaction of the solar wind with a completely unmagnetized Earth. When the solar wind encounters unmagnetized objects, such as Venus (Luhmann 1995) and comets (Konz et al. 2004), magnetic barriers and ionopauses develop. Although the interaction of a fully ionized and a weakly ionized gas is very complex, an important characteristic can be identified - the generation of magnetic fields caused by relative plasma-neutral gas shear flows. It has been shown (Huba \& Fedder 1993) that this process operates in the Venus' ionosphere and is responsible for the nondipole magnetic field measured there. The same process has been studied in detail for the generation of seed magnetic fields in emerging galaxies (Wiechen et al. 1998; Birk et al. 2002) and in circumstellar disks (Birk et al. 2003). A kinematic estimate indicates that relatively strong magnetic fields are generated in the Earth's ionosphere. So far, the only way to study the dynamical non-linear interaction of the magnetized fully ionized solar wind plasma and the partially ionized Earth's ionosphere are three-dimensional plasma-neutral gas simulations. 
Our numerical studies show the draping of the magnetic field of the solar wind and the self-generation of a relatively strong magnetic field in the Earth's ionosphere.

\section{A kinematic estimate of the ionospheric magnetic field induced by the solar wind}

The interaction of a fully ionized plasma with a partially ionized gas can be described by the fluid balance equations for the mass densities, momentum densities and thermal pressures of the different species (see Sect. 3) together with the generalized Ohm's law. Ohm's law connects the electric fields and electric currents in a plasma. For the low-frequency dynamics we are interested in, it can be deduced from the inertialess electron momentum equation (Mitchner \& Kruger 1973; Wiechen et al. 1998)

$$
\begin{aligned}
0= & -\nabla p_{\mathrm{e}}-n_{\mathrm{e}} e\left(\boldsymbol{E}+\frac{1}{c} \boldsymbol{v}_{\mathrm{e}} \times \boldsymbol{B}\right)-\rho_{\mathrm{e}} v_{\mathrm{ei}}\left(\boldsymbol{v}_{\mathrm{e}}-\boldsymbol{v}_{\mathrm{i}}\right) \\
& -\rho_{\mathrm{e}} v_{\mathrm{en}}\left(\boldsymbol{v}_{\mathrm{e}}-\boldsymbol{v}_{\mathrm{n}}\right)
\end{aligned}
$$

where $\boldsymbol{E}$ and $\boldsymbol{B}$ are the electric and magnetic fields, respectively, and by $p_{\mathrm{e}}, n_{\mathrm{e}}, \rho_{\mathrm{e}}, v_{\alpha}$, and $v_{\alpha \beta}$ the electron pressure, particle density, mass density, bulk velocities of the different plasma components (electrons, ions, and neutrals) and collision frequencies are denoted. The constants $e$ and $c$ are the elementary charge and speed of light. The electron velocity $\boldsymbol{v}_{\mathrm{e}}$ can be written in the form $\boldsymbol{v}_{\mathrm{e}}=\boldsymbol{v}-m_{\mathrm{i}} \boldsymbol{j} / e \rho$ with the plasma mass density $\rho=\rho_{\mathrm{e}}+\rho_{\mathrm{i}} \approx \rho_{\mathrm{i}}$, the plasma bulk velocity $\boldsymbol{v}=\left(\rho_{\mathrm{e}} \boldsymbol{v}_{\mathrm{e}}+\rho_{\mathrm{i}} \boldsymbol{v}_{\mathrm{i}}\right) / \rho \approx \boldsymbol{v}_{\mathrm{i}}$ and the electric current density $\boldsymbol{j}=n e\left(\boldsymbol{v}_{\mathrm{i}}-\boldsymbol{v}_{\mathrm{e}}\right)\left(n=n_{\mathrm{e}}=n_{\mathrm{i}}\right.$ is the particle density of the quasi-neutral plasma). Consequently, the inertialess equation of motion of the electrons yields Ohm's law in the form

$$
\begin{aligned}
\boldsymbol{E}+\frac{1}{c} \boldsymbol{v} \times \boldsymbol{B}= & \frac{1}{n e} \nabla p_{\mathrm{e}}+\frac{1}{4 \pi n e}(\nabla \times \boldsymbol{B}) \times \boldsymbol{B} \\
& -\frac{1}{n e}\left[\rho_{\mathrm{e}} v_{\mathrm{ei}}\left(\boldsymbol{v}_{\mathrm{e}}-\boldsymbol{v}_{\mathrm{i}}\right)-\rho_{\mathrm{e}} v_{\mathrm{en}}\left(\boldsymbol{v}_{\mathrm{e}}-\boldsymbol{v}_{\mathrm{n}}\right)\right]
\end{aligned}
$$

where Ampère's law has been used to eliminate the current density. By Faraday's law one obtains an equation that governs the dynamical evolution of the magnetic field

$$
\begin{aligned}
\frac{\partial \boldsymbol{B}}{\partial t}= & \nabla \times(\boldsymbol{v} \times \boldsymbol{B})-\frac{c}{e} \nabla \times\left(\frac{\nabla p_{\mathrm{e}}}{n}\right)-\nabla \times(\eta \nabla \times \boldsymbol{B}) \\
& -\frac{c}{4 \pi e} \nabla \times\left(\frac{(\nabla \times \boldsymbol{B}) \times \boldsymbol{B}}{n}\right) \\
& -\frac{c m_{\mathrm{e}}}{e} \nabla \times\left[v_{\mathrm{en}}\left(\boldsymbol{v}_{\mathrm{e}}-\boldsymbol{v}_{\mathrm{n}}\right)\right]
\end{aligned}
$$

with the magnetic diffusivity $\eta=c^{2} v_{\mathrm{ei}} m_{\mathrm{e}} / 4 \pi n e^{2}$. The final term, that results from the collisional momentum transfer between electrons and neutrals due to shear flow, is crucial for the generation of the magnetic fields under investigation. The collision frequency $v_{\mathrm{en}}$ is given by (Huba 1998) $v_{\mathrm{en}}=$ $n_{\mathrm{n}} \sigma\left(k T_{\mathrm{e}} / m_{\mathrm{e}}\right)^{1 / 2}$ with a scattering cross section of $\sigma \simeq 4 \times$ $10^{-15} \mathrm{~cm}^{-2}$ and $k, T_{\mathrm{e}}$ denoting the Boltzmann constant and the electron temperature, respectively. The main interaction between the solar wind and the Earth's ionosphere is expected to happen at a ionospheric height of about $350 \mathrm{~km}$ where the ram pressure of the solar wind with an average velocity of $v_{\mathrm{SW}}=450 \mathrm{~km} \mathrm{~s}^{-1}$ is balanced by the ionospheric pressure. The collision frequency of solar wind electrons with ionospheric neutrals can be estimated to be $v_{\mathrm{en}} \approx 200 \mathrm{~Hz}$. The solar wind consists of a fully ionized plasma that carries along a magnetic field of about $B_{\mathrm{SW}} \approx 2 \times 10^{-4} \mathrm{G}$ (see, e.g., Priest 1987 for the solar wind parameters). The shear length scale $L$, that defines an estimate for the nabla operator in the final term in Eq. (3), is the least known parameter in our analysis. In fact, we scale our numerical results to this (free) parameter. Shear lengths of $L=10 \mathrm{~km}$ seem to be reasonable. The ionospheric height gives an upper limit for $L$. The order of magnitude of the generated magnetic fields in the rest frame of the neutrals can roughly be estimated from the final term of Eq. (3) as

$$
\begin{aligned}
B_{\text {gen }} & \approx \tau v_{\mathrm{en}} \frac{m_{\mathrm{e}} c}{e} \frac{v_{\mathrm{SW}}}{L} \\
& =5 \times 10^{-4} \mathrm{G}\left[\frac{v_{\mathrm{en}}}{200 \mathrm{~Hz}}\right]\left[\frac{v_{\mathrm{SW}}}{450 \mathrm{~km} / \mathrm{s}}\right]\left[\frac{L}{10 \mathrm{~km}}\right]^{-1} \tau
\end{aligned}
$$

where $\tau$ is the generation time scale. The time scale of the diffusion of the magnetic field measured by $\eta$ is much larger than $\tau$. An upper limit for $\tau$ cannot be given from a pure kinematic consideration. The saturation magnetic field results from self-consistent simulations of the entire dynamics presented in Sect. 3.

For the parameters given, a field strength comparable to the present dipole value is generated after only ten minutes in the ionosphere. Thus, magnetic fields can be generated very efficiently around the unmagnetized Earth.

\section{The numerical model}

The interaction of the solar wind with the Earth's ionosphere can be modeled by a plasma-neutral gas two fluid description. In our simulations, the following normalized plasma-neutral gas equations are numerically integrated

$$
\frac{\partial \rho}{\partial t}=-\nabla \cdot(\boldsymbol{v} \rho)
$$

$$
\begin{aligned}
& \frac{\partial \rho_{\mathrm{n}}}{\partial t}=-\nabla \cdot\left(\boldsymbol{v}_{\mathrm{n}} \rho_{\mathrm{n}}\right) \\
& \frac{\partial}{\partial t}(\rho \boldsymbol{v})=-\nabla \cdot(\rho \boldsymbol{v} \boldsymbol{v})-\nabla p+(\nabla \times \boldsymbol{B}) \times \boldsymbol{B}-\rho v_{\mathrm{pn}}\left(\boldsymbol{v}-\boldsymbol{v}_{\mathrm{n}}\right)
\end{aligned}
$$

$$
\frac{\partial}{\partial t}\left(\rho_{\mathrm{n}} \boldsymbol{v}_{\mathrm{n}}\right)=-\nabla \cdot\left(\rho_{\mathrm{n}} \boldsymbol{v}_{\mathrm{n}} \boldsymbol{v}_{\mathrm{n}}\right)-\nabla p_{\mathrm{n}}-\rho_{\mathrm{n}} v_{\mathrm{np}}\left(\boldsymbol{v}_{\mathrm{n}}-\boldsymbol{v}\right)
$$

$$
\begin{aligned}
\frac{\partial \boldsymbol{B}}{\partial t}= & \nabla \times(\boldsymbol{v} \times \boldsymbol{B})-\nabla \times(\eta \nabla \times \boldsymbol{B})-\nabla \times\left[\hat{\mathrm{e}}_{\mathrm{en}}\left(\boldsymbol{v}_{\mathrm{n}}-\boldsymbol{v}\right)\right] \\
\frac{\partial p}{\partial t}= & -\boldsymbol{v} \cdot \nabla p-\gamma p \nabla \cdot \boldsymbol{v}+(\gamma-1)\left(2 \eta(\nabla \times \boldsymbol{B})^{2}\right. \\
& \left.-3 v_{\mathrm{pn}}\left(p-\frac{\rho}{\rho_{\mathrm{n}}} p_{\mathrm{n}}\right)+\rho v_{\mathrm{pn}}\left(\boldsymbol{v}-\boldsymbol{v}_{\mathrm{n}}\right)^{2}\right)
\end{aligned}
$$




$$
\begin{aligned}
\frac{\partial p_{\mathrm{n}}}{\partial t}= & -\boldsymbol{v}_{\mathrm{n}} \cdot \nabla p_{\mathrm{n}}-\gamma_{\mathrm{n}} p_{\mathrm{n}} \nabla \cdot \boldsymbol{v}_{\mathrm{n}} \\
& +\left(\gamma_{\mathrm{n}}-1\right)\left(3 v_{\mathrm{np}}\left(p_{\mathrm{n}}-\frac{\rho_{\mathrm{n}}}{\rho} p\right)+\rho_{\mathrm{n}} v_{\mathrm{np}}\left(\boldsymbol{v}_{\mathrm{n}}-\boldsymbol{v}\right)^{2}\right)
\end{aligned}
$$

where $p=p_{\mathrm{e}}+p_{\mathrm{i}}$ and $\gamma$ denote the thermal plasma pressure and the ratio of specific heats (all quantities are now to be taken as dimensionless). The index " $n$ " identifies the neutral gas quantities. The effective plasma-neutral gas collision frequency is $v_{\mathrm{pn}}=\left(m_{\mathrm{e}} v_{\mathrm{en}}+m_{\mathrm{i}} v_{\mathrm{in}}\right) /\left(m_{\mathrm{e}}+m_{\mathrm{i}}\right)$ where $v_{\mathrm{pn}} \rho=v_{\mathrm{np}} \rho_{\mathrm{n}}$ holds to guarantee momentum conservation. In the induction equation (Eq. (9)), $\hat{v}_{\mathrm{en}}$ denotes the normalized expression for $v_{\mathrm{en}} c m_{\mathrm{e}} / e$. The final term in the induction equation results in self-magnetization caused by relative sheared plasma-neutral gas flows (Lesch et al. 1989; Huba \& Fedder 1993; Wiechen et al. 1998). Normalization of the physical quantities is carried out by the typical plasma mass density, the typical length scale, the plasma Alfvén speed and time scale. The thermal pressure is normalized to the magnetic pressure. The thermal pressure is measured by a typical number density and the kinetic temperature. The source terms in the balance equations for the mass densities, momenta and thermal pressures are treated in a flux-corrected manner (Birk \& Otto 1996). For the simulation shown, we use a numerical domain of 7 Earth radii around the Earth in each direction (with the exception of 3 Earth radii in the solar direction) and one million grid points. The Earth is located at $x=y=z=0$, the solar wind enters the numerical box from the upper $y$-boundary. At all the other boundaries outflow conditions are chosen. The neutral gas of the ionospheric layer around the Earth shows a cosh ${ }^{-2}$-decay toward the interplanetary space.

\section{Numerical results: Draping and self-magnetization}

Figure 1 shows an arrow plot of the solar wind velocity field at three different times ( $t=30 \mathrm{~s}$; upper plot, $t=60 \mathrm{~s}$; middle plot and $t=600 \mathrm{~s}$; lower plot). The wind encounters the Earth and is deflected around the planet.

The magnetic field lines carried by the solar wind are draped around the Earth (Fig. 2). The draping leads to an amplification of the magnetic field near the Earth by one order of magnitude. This effect is well known, e.g., from investigations on the interaction of the solar wind with the unmagnetized Venus (Russel 1993; Luhmann 1995).

Close to the Earth, the momentum transfer between the charged particles of the solar wind and the neutrals of the Earth's ionosphere becomes important (see final terms in Eqs. (7) and (8)). Consequently, a new strong non-dipole magnetic field is generated by the sheared relative plasma-neutral gas motion (see final term in Eq. (9)). Parameter studies show the kinematic finding (see Sect. 2) that the strength of the generated magnetic field depends on the shear length $L$. The maximum shear length is fixed in the simulation by an appropriate choice of the profile for $\hat{v}_{\mathrm{en}}$. For a shear length $L=10 \mathrm{~km}$ a magnetic field of about the present dipole strength $\left(B_{\max } \approx\right.$ $0.3 \mathrm{G}$ ) is induced in the ionosphere after about $10 \mathrm{~min}$ (Fig. 3). For a given $L$ the time scale of the field generation $\tau$ results
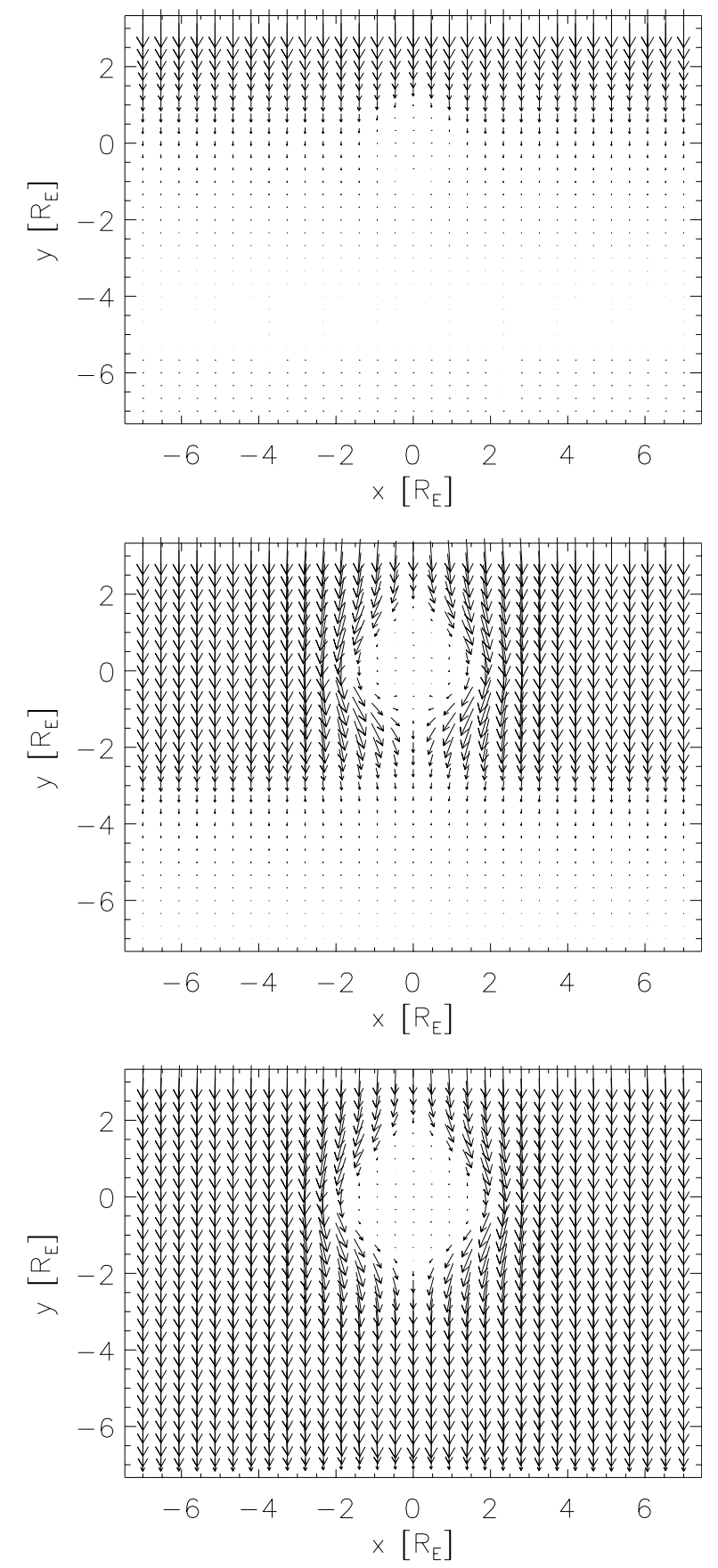

Fig. 1. The solar wind flow around the Earth in the $z=0$-plane at $t=30 \mathrm{~s}$ (upper plot), $t=60 \mathrm{~s}$ (middle plot) and $t=600 \mathrm{~s}$ (lower plot $)$.

from the dynamics. The field is generated in heights of some hundreds of kilometers all around the Earth with the exception of the subsolar region where the magnetic field is weaker. If the shear length were chosen as say $100 \mathrm{~km}$, the maximum of the generated field strength would be $B_{\max } \approx 0.03 \mathrm{G}$.

We find that the draping effect is much weaker than the magnetic field self-generation by the shear flow.

\section{Conclusions}

We studied the interaction of the magnetized fully ionized solar wind plasma with the unmagnetized partially ionized Earth's 


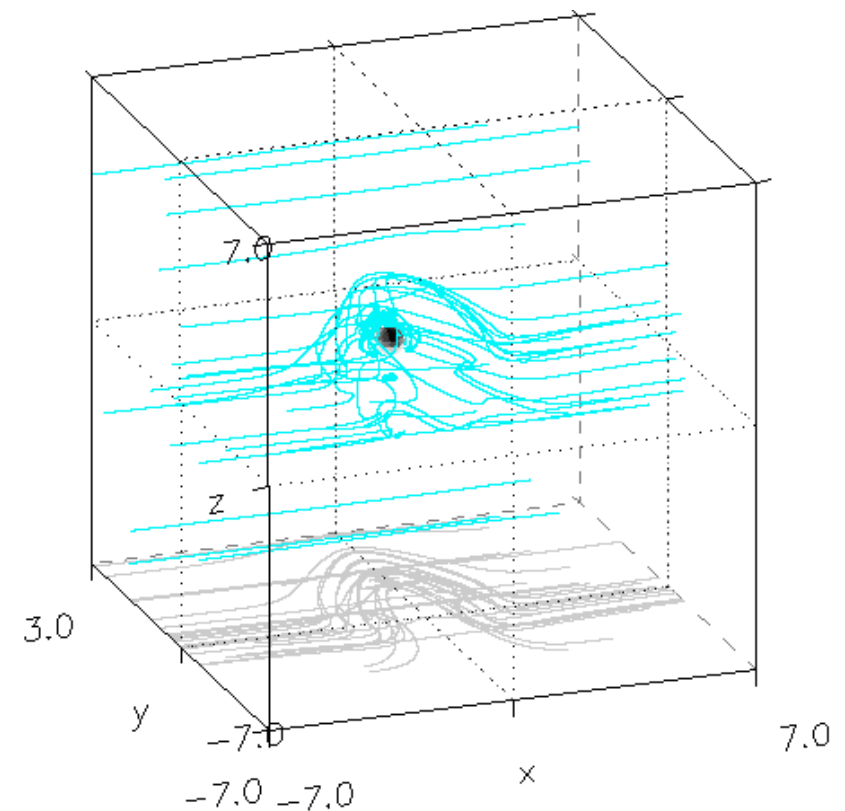

Fig. 2. The draping of the magnetic field lines of the solar wind around the Earth at $t=600 \mathrm{~s}$.

ionosphere. When the solar wind hits the Earth the magnetic field lines carried along with it are draped around the planet. What is more important, the relative motion between the solar wind plasma and the ionosphere results in the self-generation of magnetic fields in the ionospheric layer. The strengths of these fields depend on the shear length of the relative flows, which, in contrast to the other relevant physical parameters, is not well known. For a reasonable shear length of $L=10 \mathrm{~km}$ the maximum strength of the newly generated magnetic field is comparable to the one of the present dipole field. Consequently, even in the case of a complete breakdown of the Earth's dynamo, the biosphere is still shielded against cosmic rays, in particular coming from the sun, by the magnetic field induced by the solar wind.

\section{References}

Birk, G. T., \& Otto, A. 1996, J. Comp. Phys., 125, 513

Birk, G. T., Wiechen, H., \& Lesch, H. 2002, A\&A, 393, 685

Birk, G. T., Wiechen, H., Kopp, A., \& Lesch, H. 2003, MNRAS, 339,

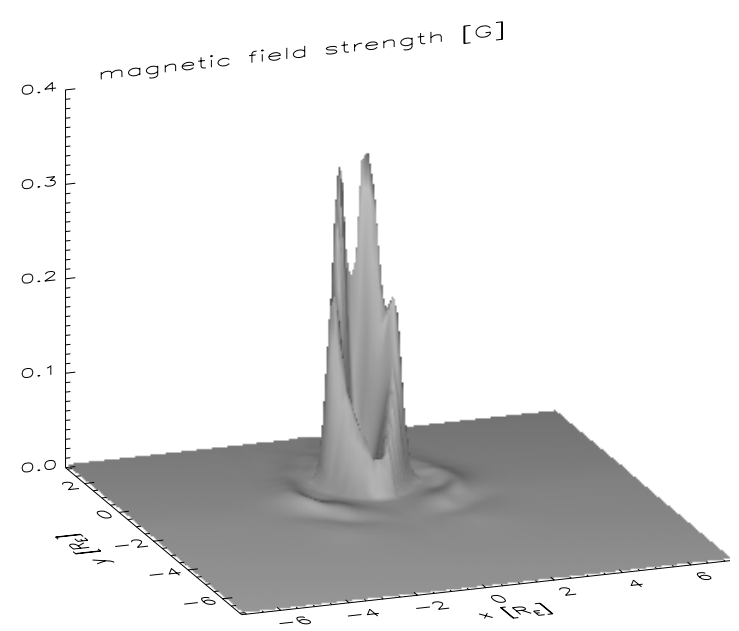

Fig. 3. The strength of the induced magnetic field in the Earth's ionosphere at $t=500 \mathrm{~s}$.

Frank, M. 2000, Phil. Trans. Soc. Lond. A, 358, 1089

Gee, J. S., Cande, S. C., Hildebrand, J. A., Donnelly, K., \& Parker, R. L. 2000, Nature, 408, 827

Guyodo, Y., \& Valet, J.-P. 1999, Nature, 399, 249

Huba, J. D., \& Fedder, J. A. 1993, Phys. Fluids B, 5, 3799

Huba, J. D. 1998, NRL Plasma Formulary, 39

Juarez, M. T., Tauxe, L., Gee, J. S., \& Pick, T. 1998, Nature, 394, 878

Konz, C., Birk, G. T., \& Lesch, H. 2004, A\&A, 415, 791

Lesch, H., Crusius, A., Schlickeiser, R., \& Wielebinski, R. 1989, A\&A, 217, 99

Luhmann, J. G., \& Bauer, S. J. 1992, in Venus and Mars: Atmospheres, ionospheres, and solar wind interactions, Washington D.C., AGU, 417

Luhmann, J. G. 1995, in Introduction to Space Physics, ed. M. G. Kivelson, \& C. T. Russell (Cambridge University Press), 203

Mitchner, M., \& Kruger, C. H. 1973, Partially ionized gases (New York: Wiley)

Priest, E. R. 1987, Solar Magnetohydrodynamics (Dordrecht: Reidel) Russel, C. T. 1993, Rep. Prog. Phys., 56, 687

Selkin, P. A., \& Tauxe, L. 2000, Phil. Trans. R. Soc. Lond. A, 358, 1065

Valet, J.-P., \& Meynadier, L. 1993, Nature, 366, 234

Valet, J.-P. 2003, Rev. Geophys., 41, 4

Wiechen, H., Birk, G. T., \& Lesch, H. 1998, A\&A, 334, 388

Wiechen, H., Birk, G. T., \& Lesch, H. 2000, Phys. Plasmas, 7, 701 